Preprint ESI 1602, TUW-05-03

\title{
A note on dilaton gravity with non-smooth potentials
}

\author{
Christian G. Böhmer* \\ *The Erwin Schrödinger International Institute for Mathematical \\ Physics, Boltzmanngasse 9, A-1090 Wien, Austria. \\ *Institut für Theoretische Physik, Technische Universität Wien, \\ Wiedner Hauptstrasse 8-10, A-1040 Wien, Austria. \\ Piotr Bronowski ${ }^{\dagger}$ \\ †Institute for Theoretical Physics, University of Lodz, \\ Pomorska 149/153, PL-90-236 Lodz, Poland.
}

\begin{abstract}
Recent interest in brane world models motivates the investigation of generic first order dilaton gravity actions, with potentials having some non-smoothness. We consider two different types of $\delta$-like contributions in the action and analyse their effects on the solutions. Furthermore a second source of non-smoothness arises due to the remaining ambiguities in the solutions in the separated smooth patches, after fixing all other constants by matching and asymptotic conditions. If moreover staticity is assumed, we explicitly construct exact solutions.

With the methods described, for example models with point like sources or brane world models (where the second source of non-smoothness becomes crucial), can now be treated as non-smooth dilaton gravity theories.

Dedicated to Wolfgang Kummer at the occasion of his Emeritierung
\end{abstract}

Keywords: $2 d$ gravity, integrable models, non-smoothness, dilaton gravity

PACS: 04.60.Kz, 11.25.Uv

\footnotetext{
*e-mail: boehmer@hep.itp.tuwien.ac.at

$\dagger$ e-mail: pbronowski@poczta.fm
} 


\section{Introduction}

In recent years brane world scenarios [1] like e.g. brane world cosmology [2] have been widely investigated as alternatives to the standard model of particle physics. In terms of the metric such models are realised by a continuous metric which has a discontinuity in the first derivative. In the action this is represented by $\delta$-like 'matter' terms. Usually the resulting gravitational field equations were analysed in the standard second order formulation, see e.g. [3,4] for a $\delta$-like 'matter' term in the second order dilaton gravity action. Here we present the necessary considerations to analyse these models in the first order formalism of dilaton gravity.

Lower and two-dimensional gravity models, cf [5-7], have become a rich source of investigation in the last two decades since they allow solutions to problems, not yet solved in the four-dimensional case, like the background independent quantisation of gravity in $1+1$ dimensions. The two-dimensional Einstein-Hilbert action is only a surface term, the Gauss-Bonnet term, hence intrinsically two-dimensional models must be supplemented by further structure since they are otherwise locally trivial. In compactifications from higher dimensions this additional structure is provided automatically by the dilaton field. For spherically reduced four-dimensional gravity the dilaton field can be regarded as the surface area of the sphere.

The resulting second order action can be written in an equivalent first order form by introducing auxiliary fields [8]. Its action in two dimensions $[7,8]$ reads

$$
\begin{aligned}
L=\int\left[X^{+}(d-\omega) \wedge e^{-}+X^{-}\right. & (d+\omega) \wedge e^{+} \\
& \left.+X d \omega+V(X) \epsilon+X^{+} X^{-} U(X) \epsilon+\mathcal{L}_{m} \epsilon\right],
\end{aligned}
$$

where $X$ is the dilaton field, $e^{a}$ is the zweibein 1-form, $\epsilon=e^{+} \wedge e^{-}$is the volume 2-form. The 1-form $\omega$ represents the spin-connection $\omega^{a}{ }_{b}=\epsilon^{a}{ }_{b} \omega$ with $\epsilon_{a b}$ being the totally skew-symmetric Levi-Civitá symbol, $\mathcal{L}_{m}$ stands for some matter Lagrangian. Note that the action (11) depends on two auxiliary fields $X^{ \pm}$.

The dilaton potential $\mathcal{V}\left(X, X^{+} X^{-}\right)$is an arbitrary function depending solely on Lorentz invariant combinations of $X$ and $X^{+} X^{-}$. All physically relevant models are covered by a special form $\mathcal{V}\left(X, X^{+} X^{-}\right)=V(X)+X^{+} X^{-} U(X)$.

In the case of spherical symmetry (or more precisely, spherical reduction of spherically symmetric four-dimensional gravity) $V(X)=-\lambda^{2}$ and $U(X)=$ $-1 /(2 X)$. The parameter $\lambda$ of mass dimension one arises as a scaling factor of the dilaton.

If $\mathcal{V}\left(X, X^{+} X^{-}\right)$contains non-smooth contributions, the classical solution for such a scenario can be obtained straightforwardly by solving the model in patches where the potential is smooth and by imposing as matching condition continuity of the Killing norm or equivalently continuity of the metric. This, together with asymptotic conditions on the line element, will fix all integration constants following standard methods (chapter 3 of [7]). 


\section{Non-smooth potentials}

The equations of motion derived from the action (1) by varying with respect to $\omega, e^{\mp}, X$ and $X^{\mp}$ are given by

$$
\begin{aligned}
& d X+X^{-} e^{+}-X^{+} e^{-}=0 \\
& (d \pm \omega) X^{ \pm} \mp \mathcal{V} e^{ \pm}=0 \\
& d \omega+\epsilon \frac{\partial \mathcal{V}}{\partial X}=0 \\
& (d \pm \omega) \wedge e^{ \pm}+\epsilon \frac{\partial \mathcal{V}}{\partial X^{\mp}}=0
\end{aligned}
$$

respectively. The matter parts $W^{ \pm}=\delta L_{m} / \delta e^{\mp}$ and $W=\delta L_{m} / \delta X$ were neglected because in this paper we will only consider matter contributions that can be incorporated into the dilaton potentials. Therefore, one may argue that matter contributions are not necessary since the sources of non-smoothness are only the dilaton potentials. However, the interpretation in terms of matter is often useful.

Linear combination of the two equations (3) with coefficients $X^{\mp}$, respectively, together with (2) yields

$$
d\left(X^{+} X^{-}\right)+X^{+} X^{-} U(X) d X+V(X) d X=0 .
$$

Multiplying this by the integrating function

$$
I(X)=c \exp \int^{X} U(y) d y
$$

one obtains the constant of motion (Casimir function)

$$
d \mathcal{C}=0, \quad \mathcal{C}=I X^{+} X^{-}+w
$$

where $w$ is defined by

$$
w(X)=\int^{X} I(y) V(y) d y
$$

The multiplicative ambiguity in the definition of the integrating factor $I(X)$ $(c \in \mathbb{R})$ has been exhibited explicitly for later convenience.

If some discontinuities in the potentials $U(X)$ and $V(X)$ is allowed, then the smoothness of all other geometrical quantities can be read off from the equations of motion (2) - (5), see table 1) We introduce the notion of a smoothness degree $n$ which refers to $C^{n}$, e.g. a continuous but non-differentiable function has smoothness degree of 0 . Only non-smoothness degrees $n, m \geq-2$ are considered, i.e. no $\delta^{\prime}$-like terms in the action will be allowed. The superscript in $\mathcal{C}^{(g)}$ indicates the conserved quantity with respect to geometry. If additional matter fields are present, the total conserved quantity is $\mathcal{C}^{(t o t)}=\mathcal{C}^{(g)}+\mathcal{C}^{(m)}$. The case where $m=n=-2$ in general is ill defined, since the integrand of 
$w$, namely $I(X) V(X)$, may not be a well defined distribution. If we require a continuous line element, at first glance, there can be only discontinuities in the dilaton potential, but no distributional contributions. However, we are still free to choose Casimir constants in each patch separately, and in this way remove such discontinuities of the metric. On the other hand, the smoothness degree in some special cases can be improved, note e.g $|x| \frac{d|x|}{d x}=x$.

\begin{tabular}{|l||c|c|}
\hline Quantity & Symbol & naive smoothness-degree \\
\hline \hline potential & $U$ & $m \in \mathbb{Z}$ \\
potential & $V$ & $n \in \mathbb{Z}$ \\
integrating factor & $I$ & $m+1$ \\
function & $w$ & $\min (m+2, n+1)$ \\
conserved quantity & $\mathcal{C}^{(g)}$ & $\min (m+2, n+1)$ \\
dual basis & $e^{ \pm}$ & $\min (m+1, n+1)$ \\
spin connection & $\omega$ & $\min (m, n)$ \\
metric & $g_{\mu \nu}$ & $\min (m+1, n+1)$ \\
curvature & $R$ & $\min (m-1, n-1)$ \\
torsion & $T$ & $\min (m, n)$ \\
\hline
\end{tabular}

Table 1: Smoothness degree of the dilaton potentials and quantities derived thereof.

For generic potentials $V(X)$ and $U(X)$ the full solution to the equations of motions derived from the action (11) reads $[7,9]$

$$
\begin{aligned}
& e^{+}=X^{+} I d f, \quad e^{-}=\frac{d X}{X^{+}}+X^{-} I d f, \\
& \omega=-\frac{d X^{+}}{X^{+}}+\mathcal{V} I d f \\
& \mathcal{C}=I X^{+} X^{-}+w=\text { const. } \\
& d s^{2}=2 I d f \otimes d X+2 I(\mathcal{C}-w) d f \otimes d f .
\end{aligned}
$$

In the following some $\delta$-like contributions $\delta\left(X-X_{0}\right)$ to the potentials $V(X)$ and $U(X)$ will be introduced:

(i) Let us start with an additional contribution to the dilaton gravity action (11) of the form $F(X) \delta\left(X-X_{0}\right)$, which can be absorbed in the dilaton potential $V(X)$, by noting $\tilde{V}(X)=V(X)+F(X) \delta\left(X-X_{0}\right)$. Without loss of generality we may assume that $X_{0}$ is positive. Together with a generic potential $U(X)$ the complete solution to the equations of motions is given by (10)-(13). The effect of such a $\delta$-like term to the potential $V(X)$ enters the solution in the absolutely conserved quantity (12) as

$$
\mathcal{C}=I(X) X^{+} X^{-}+\tilde{w}(X),
$$


where

$$
\begin{aligned}
\tilde{w}(X) & =\int^{X} I(y) V(y) d y+\int^{X} F(y) \delta\left(y-X_{0}\right) d y \\
& = \begin{cases}w(X)+F\left(X_{0}\right), & X>X_{0}, \\
w(X), & X<X_{0} .\end{cases}
\end{aligned}
$$

For a continuous line element (13) at $X=X_{0}$ the two Casimir constants $\mathcal{C}_{X>X_{0}}$ and $\mathcal{C}_{X<X_{0}}$ of the respective patches $X>X_{0}$ and $X<X_{0}$ are related by $\mathcal{C}_{X>X_{0}}=\mathcal{C}_{X>X_{0}}+F\left(X_{0}\right)$. So the matching condition, applied to the Killing norm, gives a shift between the two Casimir constants in the two different patches. Note that in this case the metric also is differentiable at $X=X_{0}$ (in fact $C^{\infty}$ ). If the metric's first derivative shall jump, this can only be achieved by introducing some non-smoothness via the relation between the dilaton and the 'physical' coordinate of the manifold, see the discussion below.

(ii) Secondly, we consider an additional term to the action of the form $X^{+} X^{-} G(X) \delta\left(X-X_{0}\right)$, which now can be absorbed in the other dilaton potential $U(X), \tilde{U}(X)=U(X)+G(X) \delta\left(X-X_{0}\right)$. This yields

$$
\begin{aligned}
\tilde{I}(X) & =c \exp \left(\int^{X} U(y)+\int^{X} G(y) \delta\left(y-X_{0}\right) d y\right) \\
& = \begin{cases}I(X) G_{0}, & X>X_{0}, \\
I(X), & X<X_{0},\end{cases}
\end{aligned}
$$

where we denoted $G_{0}=\exp \left(G\left(X_{0}\right)\right)$ and where $\tilde{w}(X)$ is given by

$$
\tilde{w}(X)=\int^{X} \tilde{I}(y) V(y) d y= \begin{cases}w(X) G_{0}, & X>X_{0}, \\ w(X), & X<X_{0} .\end{cases}
$$

The motivation of the term $X^{+} X^{-} G(X) \delta\left(X-X_{0}\right)$ comes from the following observation. $\delta$-like contributions in the equivalent second order formulation of dilaton theories [7] are usually of the form $\delta\left(r-r_{0}\right)$ which with $X=X(r)$ can be written as $\delta\left(r-r_{0}\right)=\left|X^{\prime}(r)\right| \delta\left(X(r)-X_{0}\right)$. Equation (2) expresses the directional derivatives of $X$ in terms of $X^{ \pm}$and therefore the term $X^{\prime}$ naturally suggests then the above mentioned form. For a discussion of distributions on general manifolds, see e.g. [10]. The term $\left|X^{\prime}\left(r_{0}\right)\right|$ can also be regarded as the Jacobian of the coordinate transformation between the dilaton $X$ and the 'physical' coordinate $r$.

As already discussed in the introduction the metric should be continuous at $X=X_{0}$. For the second case this yields $\mathcal{C}_{X<X_{0}}=\mathcal{C}_{X>X_{0}} G_{0}-w\left(X_{0}\right)\left(1-G_{0}^{2}\right)$. In contrast to the previous case the metric is not differentiable at the point $X=X_{0}$.

To specify the gauge of the metric to Eddington-Finkelstein form, we introduce the new variable $r$ by $d X=I^{-1} d r$,

$$
d s^{2}=2 d f \otimes d r+2 I(\mathcal{C}-w) d f \otimes d f .
$$


This coordinate redefinition may be an additional source of non-smoothness of the metric. With the help of conformal transformations, one can always eliminate the potential $U$, i.e. get a conformally related dilaton gravity model with $U=0$, (cf [7]), which of course corresponds to a different theory.

In this case the integrating factor $I$ is a constant, therefore one should have a closer look at these situations. The coordinate $r$ and the dilaton $X$ are then related by $X=I^{-1} r+X_{0}$, where $X_{0}$ is a constant of integration. This relation allows the situation $X=0$ for $r=-I X_{0}$. The simplest way to avoid such singular points is the change of the sign of the constant $I$. More specifically:

Patch "+" : In the patch $r>0$ we choose the positive sign, and put $c=1$ (which is the standard choice), so that $X>X_{0}$, and therefore we get

$$
X=I^{-1} r+X_{0}, \quad r>0 .
$$

Patch "_" : In the patch $r<0$, using the ambiguity in the definition of the integrating factor $I$, we choose the negative sign, i.e. $c=-1$ in (7). In this way also in this patch the dilaton is positively defined, $X>X_{0}$, namely

$$
X=-I^{-1} r+X_{0}, \quad r<0 .
$$

Let us moreover remark that non-smooth potentials may lead to line elements which are smooth as a function of the dilaton (case (i)), but non-smooth with respect to the 'physical' coordinate, because of the non-smooth relation between $X$ and $r$. This feature is already known in dilaton gravity models, see e.g. the "kink" discussion in [11]. A similar situation occurs with Rosen versus Brinkmann coordinates with PP-waves, (cf e.g. [12]).

\section{Example: a static point-like source}

In the recent paper of Melis and Mignemi on "Two-dimensional static black holes with point-like sources" [4] a very special dilaton gravity model is considered, supplemented with a matter part $\mathcal{L}_{m}=2 \kappa^{2} X m \delta\left(r-r_{0}\right)$. Such a matter contribution in the action is covered by the above analysis of case (ii). With the specified potentials $V(X)=-l^{2} X^{h}$ and $U(X)=0$ we find

$$
\tilde{I}(X)= \begin{cases}c G_{0} & X>X_{0} \\ c & X<X_{0}\end{cases}
$$

For case (ii) this yields

$$
\tilde{w}(X)= \begin{cases}-l^{2} c G_{0}\left(X^{h+1}-X_{0}^{h+1}\right) /(h+1) & X>X_{0}, \\ -l^{2} c\left(X^{h+1}-X_{0}^{h+1}\right) /(h+1) & X<X_{0} .\end{cases}
$$


Choosing the dilaton to be positive, which is equivalent to considering the two patches described above $(c= \pm 1)$ for positive and negative $r$ respectively, the Killing norms in the respective patches are

$$
K_{ \pm}(X)= \pm 2 G_{0}\left(\mathcal{C}_{ \pm} \pm \frac{G_{0} l^{2}}{h+1}\left(X^{h+1}-X_{0}^{h+1}\right)\right)
$$

where the upper and lower sign correspond to patch + and - respectively. The continuity of the metric at $X_{0}$ yields the matching condition $\mathcal{C}= \pm \mathcal{C}_{ \pm}$. Hence, the Killing norm reads

$$
K(X)=2 G_{0}\left(\mathcal{C}+\frac{G_{0} l^{2}}{h+1}\left(X^{h+1}-X_{0}^{h+1}\right)\right) .
$$

Comparing the latter with the result of [4], where

$$
K(X)=\frac{1}{h+1}\left(X^{h+1}-\frac{h+3}{2}\left(\frac{\kappa^{2} m}{l}\right)^{\frac{h+1}{h}}\right),
$$

simply yields

$$
G_{0}^{2}=\frac{1}{2 l^{2}}, \quad \mathcal{C}=-\frac{\sqrt{2}}{4}\left(\kappa^{2} m\right)^{\frac{h+1}{h}} l^{-\frac{1}{h}},
$$

where we put $X_{0}=\left(\kappa^{2} m / l\right)^{1 / l}$. This shows that the model considered in [4] belongs to the general class of dilaton gravity models with non-smooth potentials.

Finally we find that the ADM mass $M_{\mathrm{ADM}}$ of [4] (cf [13-15]) and the conserved quantity $\mathcal{C}$ are related by

$$
M_{\mathrm{ADM}}=-\frac{1}{\sqrt{2}} \frac{h+3}{h+1} \mathcal{C} .
$$

One has to be careful with (29) for general $h$. Strictly speaking the definition of the ADM mass requires asymptotic flatness, for $h=0$ the spacetime is indeed flat. But for $h=1$ these solutions have constant curvature. A canonical mass definition in the context of two-dimensional dilaton gravity models can be found in the appendix $\mathrm{A}$ of [16], where the relation between the conserved quantity $\mathcal{C}$ and the mass for different models is discussed.

\section{Final remarks and outlook}

We have shown how $\delta$-like terms in the first order dilaton gravity action can be dealt with. The described method was applied to a static point-like source term, where it was possible to recover previous results as a special class of generic actions with non-smooth potentials.

Let us remark that the effective line element of the virtual black hole (VBH) $[17$, 18] contains $\delta$-like contributions, which were not covered in the present work 
due to the assumption of a continuous line element. The Ricci scalar of VBHs contains $\delta$ and $\delta^{\prime}$ terms which however yield a vanishing Einstein-Hilbert action.

Non-smooth line elements are also well known in the classical theory of general relativity, e.g. thin shells $[19,20]$ are not smooth either. The same is also true for constant density perfect fluid solutions, where the energy-momentum tensor is not continuous at the boundary of the stellar object and hence the metric is of type $C^{1}$, which cannot be improved.

The first order formalism can also be applied to brane world scenarios, as was already outlined in the introduction. The metric of the simplest brane spacetime [2] is given by

$$
d s^{2}=e^{-2 k|y|} \eta_{\mu \nu} d x^{\mu} d x^{\nu}-d y^{2},
$$

where the brane is located at $y=0$ and $\eta_{\mu \nu}=\operatorname{diag}(+1,-1,-1, \ldots)$. From the dilaton gravity point of view it is natural to choose the dilaton to be $X(y)=\exp (-k|y|)$, where the non-smoothness of the metric is due to the relation between the dilaton and the two-dimensional coordinate (motivated by the above case (ii)). With the potentials $U=-1 / X$ and $V=0$ in action (1) and taking the negative sign of the integrating factor for $y>0$ and the positive one for $y<0$ into account, we recover the above metric (30) from the general solution (10)- (13).

More general brane worlds are those, where for example the geometry induced on the brane is homogeneous and isotropic, hence compatible with the cosmological principle [2], or where the induced geometry is spherically symmetric, e.g. [21,22]. These can also be analysed with the methods described if the dilaton potentials are chosen appropriately.

We hope that the present formulation will be of help in particular in the analysis of dynamical models like the scattering of a black hole and a brane or a dynamical many brane scenario, where for example two branes collapse.

\section{Acknowledgement}

We deeply thank D. Grumiller for suggestions regarding the manuscript and for useful discussions. Moreover we thank H. Balasin and D. Vassilevich for the useful discussions.

The work of CGB was supported by the Junior Research Fellowship of The Erwin Schrödinger International Institute for Mathematical Physics.

\section{References}

[1] L. Randall and R. Sundrum. An alternative to compactification. Phys. Rev. Lett., 83:4690-4693, 1999.

[2] P. Brax, C. van de Bruck, and A.-C. Davis. Brane world cosmology. Rept. Prog. Phys., 67:2183-2232, 2004. 
[3] R. B. Mann, A. Shiekh, and L. Tarasov. Classical and quantum properties of two-dimensional black holes. Nucl. Phys., B341:134-154, 1990.

[4] M. Melis and S. Mignemi. Two-dimensional static black holes with pointlike sources. 2004. gr-qc/0411136.

[5] J. D. Brown. Lower Dimensional Gravity. World Scientific, 1988.

[6] S. Nojiri and S. D. Odintsov. Quantum dilatonic gravity in $\mathrm{d}=2,4$ and 5 dimensions. Int. J. Mod. Phys., A16:1015-1108, 2001.

[7] D. Grumiller, W. Kummer, and D. V. Vassilevich. Dilaton gravity in two dimensions. Phys. Rept., 369:327-429, 2002.

[8] P. Schaller and T. Strobl. Poisson structure induced (topological) field theories. Mod. Phys. Lett., A9:3129-3136, 1994.

[9] Thomas Klösch and Thomas Strobl. Classical and quantum gravity in (1+1)-dimensions. Part I: A unifying approach. Class. Quant. Grav., 13:965-984, 1996.

[10] H. Balasin and H. Nachbagauer. The energy-momentum tensor of a black hole, or what curves the Schwarzschild geometry? Class. Quant. Grav., 10:2271-2278, 1993.

[11] D. Grumiller and W. Kummer. The classical solutions of the dimensionally reduced gravitational Chern-Simons theory. Ann. Phys., 308:211-221, 2003.

[12] P. C. Aichelburg and H. Balasin. Generalized symmetries of impulsive gravitational waves. Class. Quant. Grav., 14:A31-A42, 1997.

[13] R. B. Mann. Conservation laws and 2-d black holes in dilaton gravity. Phys. Rev., D47:4438-4442, 1993.

[14] W. Kummer and P. Widerin. Conserved quasilocal quantities and general covariant theories in two-dimensions. Phys. Rev., D52:6965-6975, 1995.

[15] W. Kummer and S. R. Lau. Boundary conditions and quasilocal energy in the canonical formulation of all $1+1$ models of gravity. Annals Phys., 258:37-80, 1997.

[16] D. Grumiller and D. Mayerhofer. On static solutions in 2d dilaton gravity with scalar matter. Class. Quant. Grav., 21:5893-5914, 2004.

[17] D. Grumiller. Virtual black hole phenomenology from 2d dilaton theories. Class. Quant. Grav., 19:997-1009, 2002.

[18] D. Grumiller, W. Kummer, and D. V. Vassilevich. Virtual black holes in generalized dilaton theories (and their special role in string gravity). European Phys. J., C30:135-143, 2003. 
[19] W. Israel. Singular hypersurfaces and thin shells in general relativity. Nuovo Cim., B44S10:1, 1966.

[20] V. A. Berezin, V. A. Kuzmin, and I. I. Tkachev. Dynamics of bubbles in general relativity. Phys. Rev., D36:2919, 1987.

[21] T. Harko and M. K. Mak. Vacuum solutions of the gravitational field equations in the brane world model. Phys. Rev., D69:064020, 2004.

[22] M. K. Mak and T. Harko. Can the galactic rotation curves be explained in brane world models? Phys. Rev., D70:024010, 2004. 